\title{
Study of Variation of Spectral Indices Derived from Hyperspectral Image on the different Crops
}

\author{
Prashant Adiver \\ Assistant Professor \\ Bannari Amman Institute of Technology \\ Sathyamangalam Tamilnadu, India
}

\begin{abstract}
Nitrogen is essential factor in monitoring growth of the crops. Nitrogen content differs from one crop to crop. Crops like soybean and corn are capable of fixing more nitrogen content compared to other crops. Hyperspectral images can be used to study variation of nitrogen content in different crops. Indian pines data set collected from Airborne invisible/infrared imaging spectrometer(AVIRIS) sensor is used the study. The image was segmented using existing ground truth data for three different type of crops corn, wheat and soybean. The paper focus on study of variation of spectral indices in three different crops namely corn, wheat and soybean. The nitrogen based spectral indices red edge index $\left(\mathrm{Cl}_{\text {red edge }}\right)$, chlorophyll green $\left(\mathrm{CI}_{\text {green }}\right)$, red edge Position (REP) was taken for the study. Since crops like soybean and corn are capable of fixing more nitrogen content they exhibit higher spectral values. The red edge index ( $\left.\mathrm{CI}_{\text {red edge }}\right)$ and chlorophyll green $\left(\mathrm{CI}_{\text {green }}\right)$ exhibited higher values in soybean and corn when compared with wheat. Red edge position (REP) exhibit still higher values in crop soybean and corn. Hence spectral indices can be used to study variations of nitrogen in three different set of crops.
\end{abstract}

\section{General Terms}

Spectral indices, Airborne, Segmented

\section{Keywords}

Hyper spectral, spectrometer, Imaging, dataset.

\section{INTRODUCTION}

Nitrogen is important content in crops. Deficiency of nitrogen can lead to stunted growth, slow growth. Monitoring of crop for nitrogen is important factor. Remote Sensing is one of the techniques to measure the properties of the crop without having any physical contact with the crop. Remote sensed data captures reflectance of radiation of sun. Researches show that remote sensing can be used to measure various Chemical components of crop like nitrogen, chlorophyll, moisture content. Remote sensing indices can also be used to measure soil properties such as moistures and minerals identification. It can also be used to study water bodies such as lakes, oceans to monitor pollution levels. In agriculture remote sensed data can be used study various diseases in the crops, identifying growing stage of the crop, predicting the yield. Remote sensed data can used to measure density of the vegetation, forest cover and deforesting activities. The Data can be used in detection of minerals. Minerals are used by geologist for mapping of minerals and high lightly properties of soil including moisture and organic content. In military the data can be used to identify military vehicles.

The electromagnetic spectrum is a collective of entire range and scope of frequencies radiation. Electromagnetic spectrum has large sets of wavelength covering from visible to infrared. Using visible light we can see objects since they corresponds to the size of the object. Light consist of photons which are having fixed with energy content which depends upon the wavelength. The human eye is able to capturing the visible region of electromagnetic spectrum in three bands (red, green, and blue), spectral images divides the spectrum into many more different set of bands. The process of dividing set of images into different bands can be extended beyond the visible region. Remote sensed data covers all the regions of electromagnetic spectrum can be classified into two types' namely Hyperspectral images and Multispectral images. A Hyper spectral image collects and processes information from across the various regions of electromagnetic spectrums. The purpose of hyper spectral imaging is to obtain the spectrum for each pixel in the image of a scene, which helps in finding objects, identifying materials, or detecting processes. Hyper spectral image is capable of capturing the wavelength even to minute resolutions .Hyper spectral images can be used to study the objects using various different wavelength Spectral imaging contains continuous spectral bands, as compared to multispectral images which measures spaced spectral bands. Multispectral images on the other hand are capable of capturing the images with the some specific wavelength across different regions of electromagnetic spectrum. The wavelengths can be separated by making use of templates or by the use of other instruments that are very sensitive to particular wavelengths. Multispectral imaging measures light in a very small number (4-15) of spectral bands. Hyper spectral images are a special case of spectral imaging where hundreds of continues spectral bands are available in Hyper spectral images, the spectrums have got very fine wavelength resolution and cover a wide range of different sets of wavelengths.

In Hyperspectral field there are two types of sensors to take images .Airborne visible/infrared imaging spectrometer (AVIRIS) is air sensor. Hyperion is a satellite based sensor. Air bourn sensors are mounted on the aircraft. NASA launched Hyperion EO-1 sensor in November 2000 which was intended to capture Hyperspectral images.Hyperion sensor has got 224 bands and spectral resolution of about $10 \mathrm{~nm}$ and spatial resolution of $30 \mathrm{~m}$.The AVIRIS is hyper spectral sensors mounted to the planes. The AVIRIS sensor was developed by NASA by jet propulsion laboratory (JPL).The sensor is able to take the image from attitude of about $20 \mathrm{~km}$ having spatial resolution of $20 \mathrm{~m}$.CASI (compact airborne spectrographic imager) is able to capture over 228 and spectral range of $400 \mathrm{~nm}$ to $1000 \mathrm{~nm}$.AISA (Airborne imaging spectrometer) has 228 bands and is capable of covering range from $430 \mathrm{~nm}$ to $1000 \mathrm{~nm}$.Hypespectral sensors also known as spectrometers are also known as spectrometers. Airborne visible sensor/infrared imaging spectrometer (AVRIS) is an airborne Hyperspectral sensor that has been operational since 1989.The sensor is having 224 continuous spectral bands each of $10 \mathrm{~nm}$ wide ranging from $400 \mathrm{~nm}$ to $2500 \mathrm{~nm}$. 
Hyperspectral image can also be used in crop diseases classification, detection and modeling .Hyper spectral image consists of 100 of narrow bands across different regions of electromagnetic spectrum. A crop reflectance of different parts of electromagnetic spectrum depends upon on crop leaf biochemical compounds and crop leaf anatomical structure. Healthy plants are capable of absorbing light in the visible range around $400-700 \mathrm{~nm}$ due to leaf photosynthesis pigments.. The percentage of light that is scattered in the nearinfrared range around $700 \mathrm{~nm}-1000 \mathrm{~nm}$ is strongly sensitive to crop leaf cell structure. Important factors influencing leaf reflectance in short-wave infrared region around $1000 \mathrm{~nm}-$ $2500 \mathrm{~nm}$ are crop leaf water and crop chemical contents. When crop undergo various types of stresses, they react through different sets of biophysical and biochemical variations such as loss of the crop leaf chlorophyll content or changes in leaf cell structures. Hyper spectral data has the advantage of detecting these slight changes in spectral reflectance of different crops. Machine learning methods can be used to develop based on these spectral reflectance values for classification of different types of crop diseases. The process usually starts with extracting features from spectral reflectance of the crop, using a classifier model prepared from diseased and healthy plant images and finally to apply the model for new data for the prediction of diseased crops, . The most common approach for feature extraction approach is to calculate different spectral Vegetation Indices (VIs) related to particular physiological parameters. Generally not able to find between healthy and diseased crops

Hyper spectral data has been widely used to successfully obtain and map the temporal and spatial variability of different crops in fields. Some researchers have evaluated the usefulness of Hyperspectral Data for estimating the yield of various types of crops. Previous studies on the application of Hyperspectral Data for yield estimation and mapping were mainly done on different sets of field crops. Few studies on the usefulness of Data of some crops have been reported. This type of study was designed to effectively evaluate the potential of airborne hyper spectral data for estimating nitrogen content in different crops.The Kjeldahl method supposed to be the standard method for the determination of nitrogen content using chemical analysis. Since because of good precision it is universally accepted, and reproducibility have made it to recognize internationally-recognized method for determining the level of Nitrogen content in crop. It also depends on destructive samplings techniques and needs follow a series of different set of procedures. This method seems to costly but also time-consuming process. The old appearance diagnostic method is very simple and fast, but it is causes some errors in nutrient status of the crops. Other method would be to estimate the nutrient status of crops from spectral images obtained during different growth stages of the crop.

Over the past few last years, several different spectral chlorophyll indices have been developed to estimate chlorophyll content both at the crop and the canopy level using Hyperspectral remote sensing data at different crop types., the good chlorophyll index should be sensitive only to chlorophyll content in crop cover region, not be changed due to soil background independent of the temporal resolution of the hyper spectral sensors, but slight is affected by atmospheric and environmental factors, the drift of the sensor radiometric and calibration, as well as light illumination geometry and senor viewing of different conditions, and not saturate in a rapid way. Such type of effects simultaneously in different measurements and at the different time of the satellite and airborne spectral images data acquisition and modification. Consequently, it is not possible to design an index which is sensitive only to the desired variable and totally not getting affected by all other parameters. However, in hyperspectral data, despite of the correction and the calibration of the different radiometric distortions the chlorophyll indices remain always affected by the artifacts caused by the optical properties in particular in a growing stage of crop growth, whether is sparse or fairly dense crop cover.

The AVIRIS data consists of 224 bands was taken for our study. Spectral indices can be derived using hyper spectral images. The spectral indices namely $\mathrm{CI}_{\text {red edge }}, \mathrm{CI}_{\text {green, }} \mathrm{REP}$ are used for our study. The red edge $\left(\mathrm{CI}_{\text {red edge }}\right)$, REP can be used to measure the nitrogen content in the crops effectively. The objective of our study is monitoring the change of the spectral indices on three different crops. Data set is segmented for three different crops namely corn, soybean, and wheat. Since soybean and corn crop are capable of fixing nitrogen contents they exhibit spectral higher spectral values when compared with wheat crop. The red edge position (REP) in the soybean exhibits higher Value when compared with other indices. Hence red edge position (REP) at $780 \mathrm{~nm}$ can be used to effectively study rate of change of nitrogen contents in different crops.

\section{RELATED WORKS}

Due Ecosystem changes occurring in the atmosphere at agriculture regions. There by it is important to monitor the farmland to any type of changes. The crop chlorophyll and nitrogen content provides information regarding physiological status of the crop. It is very important to assess the content carefully. The crop nitrogen content is directly linked to the crop chlorophyll content. Crop nitrogen content depends upon nutrients available and crop nitrogen uptake. The nitrogen content keeps changes over different stages of its development and when the crop is exposed to different types of stress. Field measurement becomes when the crop is distributed over the wide region. In order to avoid time consuming destructive estimation methods it is better to use handled device such as SPAD meter. SPAD readings are one of non-destructive and can be dependable but still it consumes a lot of time to over wide range of regions. On the other hand making use of the remote sensing method can be good estimator of chlorophyll and nitrogen content. As a result, remote sensing acts as important tool to find missing and in irrelevant information for good agricultural practices.

In recently, Zhu et al. [2] was able to detect and classify the diseased tobacco leaves using hyper spectral image. Hulk Cetin[3] reported that the vegetation index NDVI is most commonly used in identifying status of crop in many Hyper spectral and multispectral images but since the index is not so much efficient, the other indices like REP, $\mathrm{CI}_{\text {red-edge, }} \mathrm{CI}_{\text {green }}$ can be used in identifying the status. Peyman [4] showed how to classify diseased leaf classification with accuracy of up to 97\% using concept K-fold testing. He also showed that we can classify the leaves into healthy and inoculated plants by extracting the feature from hyper spectral images using classifiers Silvia Valero[5] reportedly identified the crops corn and soybean in a early growing stages .Experimental results was able to detect some of the challenges involved in detection and identification of crops. The soybean and corn spectra were taken for the study. The green color was used for soya bean and corn was plotted in red color. The difference was found in near infrared region. In this way it was possible to separate the Hyperpectral data depending upon the crop identified. 
Silvia Valero[5] is able to identify the crops using NDVI time series which is other approach. The graphs of corn and soybean were plotted using different colors. Soybean crops shows higher NDVI values in there mature stage when compared with corn crop and thereby Hyper spectral image can be used in crop in there mature stage.

Hrishikesh[6] reported that soybean crop can be easily identify from the weed and to determine moisture stress with given vegetation species using feature extraction techniques like discrete wavelet transform.. Xujun Yea[11] reported used Hyperspectral data in estimation of fruit yield in citrus. The paper shows that using vegetation indices(Vis) and newly derived two band vegetation index(TBVI) and finally by using partial least square regression technique(PLS) factors obtained by chemometric analysis. The study confirmed Hyperspectral data can be used to successfully predict the fruit yield $\mathrm{n}$ citrus.

\subsection{Methodology}

The Indian pines data set is used for our study. The scene was captured by AVIRIS sensor over Indian pine site in north western Indiana and consists of 145 times 145 pixels and 224 spectral reflectance bands in wavelength of range 0.4-2.5 $10^{\wedge}(-6)$ meters. The scene consists of two-third of agriculture, one third of agricultural. The image is taken in month of June some crops like corn, soybean are present. The ground truth available is divided into 16 classes. The bands have been reduced to 200 by removing bands covering water absorption. The following picture shows Indian pines image data set. The image is loaded in MATLAB using load command and is segmented using available ground truth data for three different set of crops namely soybean, corn and wheat. The ground truth data is shown in figure 1, Indian pines set in figure. 2 and segmented image shown in figure .3

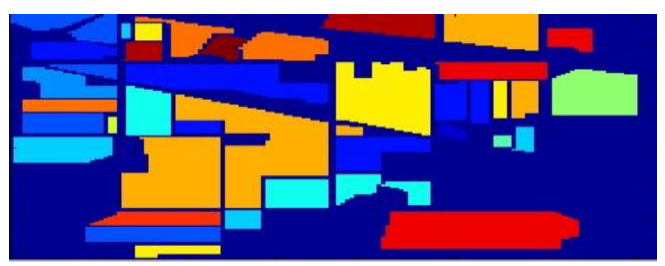

Fig 1: ground truth

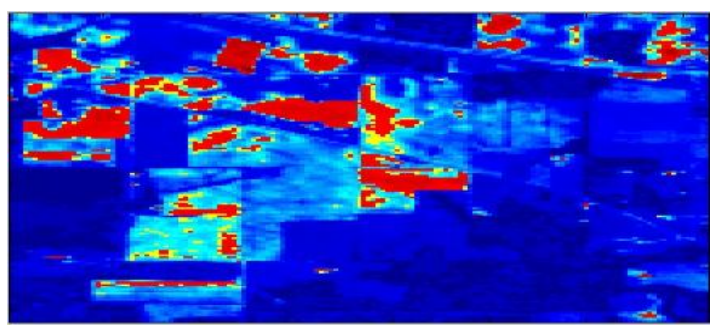

Fig 2: Indian Pine dataset

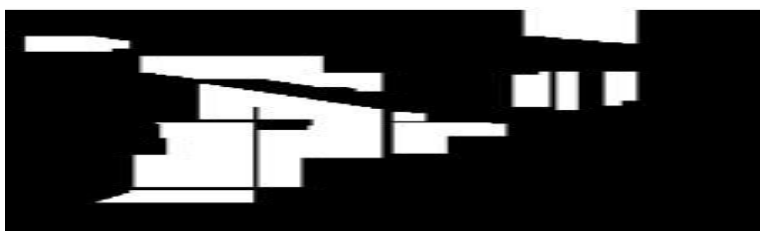

Fig 3: segmented image

\section{RESULTS}

Spectral indices can reveal lot of information when studied. The random pixel is selected from the image and reflectance values are obtained by using the available data set. The hyper spectral band starts from 400nm and continuous till 2500nm.It covers entire band starting from NIR, visible and infrared. The index REP (Red edge position) is extracted at the band 670, $780,700, \mathrm{CI}_{\text {red edge }}$ (cholorphyll red edge) around 780,710 and $\mathrm{CI}_{\text {green }}$ (cholorphyll green)around 550,780[2].The formulas needed for calculating the indices are given in the following table 1 . The results are tabulated in the table 2

TABLE 1: Formulas for calculating indices

\begin{tabular}{|l|c|c|}
\hline INDEX & FORMULATION & $\begin{array}{l}\text { REFERE } \\
\text { NCE }\end{array}$ \\
\hline REP & $\begin{array}{l}700+40 *\left(R_{670}+R_{780}\right. \\
) / 2-R_{700}\end{array}$ & {$[2]$} \\
\hline $\mathrm{CI}_{\text {red edge }}$ & $\left(\mathrm{R}_{780} / \mathrm{R}_{710}\right)-1$ & {$[2]$} \\
\hline $\mathrm{CI}_{\text {greem }}$ & $\left(\mathrm{R}_{780} / \mathrm{R}_{550}\right)-1$ & {$[2]$} \\
\hline
\end{tabular}

TABLE 2: calculated values

\begin{tabular}{|l|l|l|l|}
\hline $\begin{array}{l}\text { Spectral } \\
\text { Indices }\end{array}$ & Corn & Soybean & Wheat \\
\hline $\begin{array}{l}\text { CI(red } \\
\text { edge) }\end{array}$ & 0.09 & 0.10 & 0.06 \\
\hline REP & 652.61 & 666.46 & 639.42 \\
\hline CI(green) & 0.09 & 0.10 & 0.06 \\
\hline
\end{tabular}

The Result indicates that soybean crop indicates higher values when compared with other crops corn wheat. Since soybean is capable of fixing more nitrogen content therefore it indicates much higher values when compared with crops. Corn crop contains slightly higher values when compared with wheat. The results conclude that spectral indices can be used to determine the variation of nitrogen content in the different crops.

\section{CONCLUSION}

Remote sensing technology is one of the non-destructive methods to estimate the chlorophyll or nitrogen content in the different crops. The technology can also be used in classification of crops and identification of weeds, fruit yield, and nutrient status, physiological status of crops. Spectral images are one of feature to implement the remote sensing technologies. Hyperspectral image is one of the type of the spectral image, many sensor like AVRIS, Hyperion can be used to capture the Hyperspectral data. Future more Hyper spectral data can be used to derive the spectral indices. Spectral indices can act as indicator to study nitrogen content, chlorophyll content variations in the different sets of crops. Spectral indices can be used effectively used to monitor nitrogen content in the crop. Spectral indices such as Red edge index, REP, $\mathrm{CI}_{\text {green }}$ are used to study the rate of change of nitrogen in the three different crops. The soybean crop exhibits higher spectral values since these crops are capable of fixing more nitrogen content when compared with other crops. In future these spectral indices can be used to estimate chlorophyll, water content in crops and disease associated with different crops.

\section{ACKNOWLEDGEMENT}

I thank all staff members of my institution for their cooperation and support. 


\section{REFERENCES}

[1].J.G.P.W Clevers and L.Kooistra ,"Using hyperspectral spectral remote sensing data for retrieving total canopy chlorophyll an nitrogen content".

[2] Xiuliang Jin, Zhenhai Li, Haikuan Feng, Xingang Xu, and Guijun Yang" Newly Combined Spectral Indices"

[3] Haluk Cetin', John'. Pafford and Tom G. Muellef "Precision Agriculture Using Hyperspectral Remote Sensing and GIS"

[4] Peyman Moghadam, Daniel Ward, Ethan Goan, Srimal Jayawardena, Pavan Sikka, Emili Hernandez "Plant Disease Detection using Hyperspectral Imaging."

[5] Silvia Valero, Pietro Ceccato, Walter E.Baethgen , and JocelynChanussot"identification of agricultural crops in early stages using remote sensing images ".

[6] Hrishikesh Tamhankar, Lori Mann Bruce, Brien Henry, David Shaw"Detection of moisture stress effects on plants using hyperspectral data"

[7]xujun yea,b, jinmeng lia, kenshi sakaib, tiejun zhaob "estimation and visualizaion of nitrogen content in citrus canopy using hyperspectral imagery".
[8]. J.G.P.W Clever and L.Kooistra,"Using hyperspectral spectral remote sensing data for retrieving canopy chlorophyll and nitrogen content".

[9].Mustafa Teke ${ }^{1}$,Husne Seda Deveci,Onur Haliloglu,Sevagi Zubydu Gurbuz,Ufuk Sakarya, Tubitak uzay, "As Shor Survey of Hyperspectral Remote Sensing Applicatio in Agriculture".

[10].Mathew A.Lee,Member,IEEE,Yanbo Huang,Haibo Yao,Member,IEEE,Steven J.Thomson Lori Mann Bruce,'Determining the effects of storage on cotton and soybean leaf samples for hyperspectral analysis"

[11].Nicholas C Coops,Marie E martin and scott V Ollinger,' Prediction of Eucalypt Foliage Nitrogen Content From Satellite-Derived Hyper spectral Data".

[12] Haluk Cetin', John'. Pafford and Tom G. Muellef "Precision Agriculture Using Hyperspectral Remote Sensing and GIS"

[13] A. Bannari , K. Staenz , D. Haboudane and K. S Khurshid "Sensitivity Analysis of Chlorophyll Indices to SoilOptical Properties Using Ground-Reflectance Data"

[14] Xiuliang Jin, Zhenhai Li, Haikuan Feng, Xingang Xu, and Guijun Yang" Newly Combined Spectral Indices"

[15]YanboHuang,Haibo Yaob,FengZhaoc,andKrishnaReddy "detection of crop herbicide injury through plant ". 